\title{
Effects of Metronidazole on Periodontal Treatment Needs
}

\author{
Walter J. Loesche, * Edgar Schmidt, * Billy A. Smith, * Edith C. Morrison, * Raul \\ Caffesse, ${ }^{\dagger}$ and Philippe P. Hujoel*
}

Periodontitis, a COMmon CAUSE of TOOTH Loss in adult populations, is an inflammatory response to the overgrowth of anaerobic organisms such as spirochetes and bacteroides and, in some cases, micro-aerophilic organisms in the subgingival plaque. In the present investigation, using a double-blind clinical design, we sought to determine whether 1 week of metronidazole treatment plus debridement of the tooth surfaces was superior to 1 week of placebo treatment plus debridement (positive control) in reducing the subsequent amount of periodontal surgery given to the patients. Thirty-nine patients were randomly assigned to either the metronidazole or placebo (positive control) groups. All patients were given the necessary scaling and root planing and were unsupervised in their usage of the medication. After the completion of this treatment, they were reexamined and it was found that the metronidazole regimen caused a significant reduction in surgical needs of about 5 teeth per patient compared to the positive control (difference before and after treatment $8.3 \pm 6.8$ teeth metronidazole versus $2.9 \pm 4.8$ positive control, $P=0.007$ ). The difference between groups was maintained during the 2 to 3 years' recall period. Metronidazole had a significant effect on the site specific reduction of spirochetes: $90 \%$ of the sites in the metronidazole group versus $64 \%$ in the positivecontrol group had a decrease in the percentage of spirochetes $(P<0.05)$. We conclude that systemic metronidazole given $250 \mathrm{mg}$ tid for 7 days in conjunction with debridement of the tooth surfaces can significantly reduce the need for periodontal surgery compared to the standard regimen which included only debridement. J Periodontol; 1991; 62:247257.

Key Words: Periodontal diseases/drug therapy; metronidazole/therapeutic use; doubleblind studies.

Periodontitis, a common cause of tooth loss in older populations, is an inflammatory response to a specific, albeit chronic, bacteriological infection associated with the overgrowth of anaerobic organisms such as spirochetes and bacteroides, ${ }^{1-5}$ and in certain instances with the micro-aerophilic organism, Actinobacillus actinomycetemcomitans. ${ }^{6-8}$ These findings indicate that treatments aimed at controlling periodontal disease should contain one or more anti-microbial components directed to the reduction, if not the elimination, of the above periodontopathic organisms. ${ }^{9}$

Conventional periodontal therapy has relied almost exclusively upon mechanical debridement of the tooth surfaces as the anti-microbial component. However, with the evidence of bacterial specificity in periodontal disease, it is possible to conceive of a treatment protocol that would include short-term usage of systemic and/or local antimi-

\footnotetext{
*Departments of Biologic and Materials Sciences and Periodontics, The University of Michigan, School of Dentistry, Ann Arbor, MI.

${ }^{+}$Formerly, University of Michigan; currently, University of Texas, Health Science Center, Department of Periodontology, Houston, TX.
}

bial agents. ${ }^{10,11}$ Shinn reported in 1962 that systemic metronidazole quickly and dramatically improved the oral condition known as acute necrotizing ulcerative gingivitis (ANUG) or trench mouth. ${ }^{12}$ As ANUG had been considered to be an anaerobic fuso-spirochetal infection, ${ }^{13}$ Shinn's results lead to the demonstration that metronidazole is a specific inhibitor of anaerobic bacteria. ${ }^{14-15}$ Subsequently, it was shown that the efficacy of metronidazole in ANUG could be associated with a significant reduction in spirochetes and in Prevotella intermedia, ${ }^{16}$ one of several blackpigmented anaerobic (Bacteroides) species found in periodontal plaques.

The similarity of the anaerobic flora found in ANUG to the anaerobic flora found in adult periodontitis (AP) ${ }^{1,4}$ suggested that metronidazole could be effective in the treatment of AP. Two double-blind studies, each using different protocols and dosages, showed that metronidazole was effective in reducing probing (pocket) depths and in increasing apparent attachment level about the most severely involved teeth. ${ }^{10,17} \mathrm{~A}$ third study showed that metronidazole plus de- 
debridement was superior to either metronidazole alone or debridement alone in improving periodontal health. ${ }^{18}$

These studies measured clinical improvement by the traditional method of reduced probing depths and an apparent gain of attachment of the teeth to the surrounding tissue. In the present investigation in addition to these measurements, we sought to determine whether metronidazole treatment plus debridement was superior to placebo treatment plus debridement in reducing the subsequent amount of periodontal surgery given to the patients.

\section{MATERIALS AND METHODS}

\section{Subjects}

Patients seen at the Graduate Periodontal Clinic and who had advanced adult periodontitis (AP) as evident by deep pocketing and bone loss about one or more teeth per jaw quadrant, and who were not pregnant, had no history of drug allergies, did not have systemic conditions that required prophylactic antibiotics for dental treatments, and were not taking medication that could influence their periodontal condition (i.e., antibiotics, nonsteroidal anti-inflammatory agents) were screened for the presence of an anaerobic spirochete infection about the most diseased tooth in each quadrant. Patients who had spirochetes averaging $20 \%$ or more of the bacteria observed in a dark-field microscopic examination in two or more of the four plaque samples were invited to participate in the double-blind study. Subjects who signed an informed consent form were assigned by an individual not involved in the study using a table of random numbers to either the metronidazole or placebo group. Entry of the patients into the study occurred over a 2 year period (1982-1984). Thirty-nine patients, 19 females and 20 males, completed the study out of 50 patients initially recruited into the study. The patients who failed to complete the experimental protocol either did not return for treatments, moved from the community, or in one case refused further treatments. No age or sex differences were present in the two treatment groups.

\section{Experimental Protocol}

All patients followed the protocol shown in Figure 1. Prior to any treatments the patients were examined by a clinician (EM) using standard procedures for the measurement of probing depth and attachment levels about five sites (the distolingual site was not examined) of each tooth in the dentition. ${ }^{19}$ A device was constructed to obtain standardized radiographs of the bone levels about the teeth and the preliminary findings employing subtraction radiography have been presented elsewhere. ${ }^{20}$ These radiographs were used by the study clinician in his evaluation of treatment needs (see below).

First Treatment Planning Session. Another clinician with 20 years experience as a periodontist (BS) examined the patient with the aid of the above clinical parameters and radiographs, and determined the number of teeth that he expected would need periodontal surgery or extraction because of the severity of the periodontal involvement. This periodontist was not aware of the treatment groups that the patients were in and he was not directly involved in their treatments. Any biases or inconsistencies that he exhibited in the determinations of surgical need would be expected to occur in both groups and to be balanced as a result of the double-blind nature of the clinical design. The criteria used to determine the need for surgery or extraction at the first treatment planning session were: 1) Probing depth and attachment levels; 2) presence or absence of bleeding or exudate; 3) root topography and nature of bony defect as evidenced by the radiographs; and 4) whether access was adequate for thorough root instrumentation. Teeth that were considered as immediate candidates for extraction were retained.

Treatment. All patients received the treatment that is standard in our graduate clinic; i.e., rigorous debridement (scaling and root planing) of the root surfaces of teeth that had $3 \mathrm{~mm}$ or more probing depth and occlusal adjustment of the teeth, if necessary. This treatment was performed by 15 graduate students and monitored by a study clinician (ES). Superimposed upon this treatment regimen was a week of unsupervised usage of either metronidazole or placebo (250 mg tid) that commenced at the first clinic visit in which the root surfaces were debrided. The metronidazole tablets were provided by Zenith Pharmaceutical (Northvale, New Jersey) and the placebo tablets were specially prepared by G.D. Searle (Chicago, IL). Three to eight clinic visits were needed to complete all scaling and root planing. Teeth that normally would have been extracted after the first treatment planning session because of a hopeless periodontal prognosis were retained and debrided. If these hopeless teeth became symptomatic during the course of treatment, they were extracted and counted as teeth lost. The experimental protocol was ethical as the placebo group was in essence a positive control group who received the best therapy available in our graduate clinic. As such this group hereafter will be referred to as the positive-control group.

Second Treatment Planning Session. Four to 6 weeks after the completion of all debridement and occlusal procedures, the patients were treatment planned by the same periodontist who performed the first treatment planning examination and again he determined the number of teeth needing periodontal surgery or teeth needing extraction.

He had access at this second treatment planning session to the patients and all radiograph and clinical measurements of probing depths and attachment levels. He did not have access to his previous treatment plan detailing the teeth he had originally recommended for periodontal surgery or extractions. Based upon his decision the patient either went 


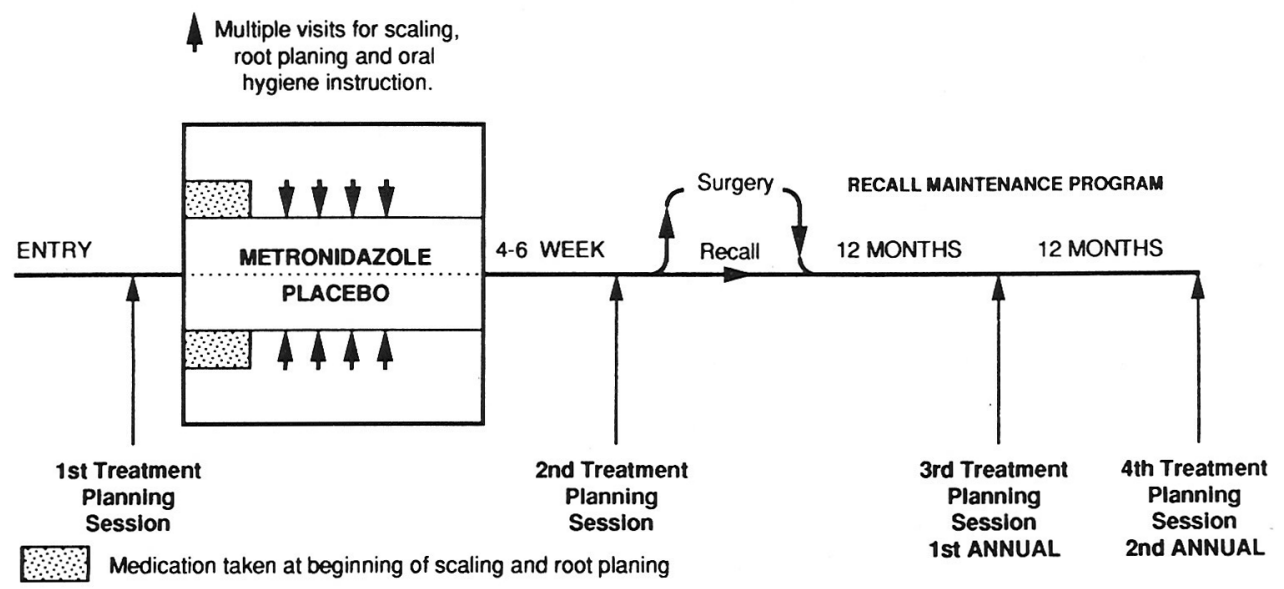

Figure 1: Patient flow through the double-blind clinical trial of the efficacy of metronidazole in periodontal disease.

directly to the recall program or went on to have periodontal surgery or extractions. This clinician also reexamined the patients at the third and fourth treatment planning sessions and, based upon his decisions, the patients received the required treatments.

The criteria used to determine the need for surgery at the second treatment planning session were: 1) Presence or absence of bleeding; 2) presence or absence of exudate; 3 ) change in probing depths; 4) root topography; 5) nature of bony defect or furcation involvement, as shown by radiographs as well as probing; 6) whether the clinician has been able to adequately scale and root plane to the extent that health has been established; (i.e., is surgical intervention necessary to gain better access for additional root instrumentation?); and 7) whether, in the judgement of the examiner, the level of health established will prevent further attachment loss. In the event that calculus or root surface roughness were present and the examiner considered the area accessible to more thorough root instrumentation, the decision relative to surgical need was delayed until the areas in question had been reinstrumented and reevaluated. If bleeding or exudate were present and probing depths were $\geq 5 \mathrm{~mm}$ then surgery was always recommended.

At the second treatment planning session, some hopeless teeth that had originally been scheduled for extraction now were judged either in need of periodontal surgery (the usual case) or not needing surgery. This beneficial effect of saving these hopeless teeth from extraction was lost in the data analysis if the saved teeth were simply moved to the surgery column. This could be illustrated by the situation in which a patient who had 18 teeth scheduled for surgery and 6 hopeless teeth scheduled for extraction at the first treatment planning session and who had 24 teeth scheduled for surgery, but no teeth scheduled for extraction at the second treatment planning session. The sum of teeth needing surgery plus extraction for both treatment planning sessions was 24 , indicating no benefits of treatment, even though 6
Table 1: Description of Tooth Status

\begin{tabular}{lcc}
\hline & $\begin{array}{c}\text { First Treatment } \\
\text { Planning Session }\end{array}$ & $\begin{array}{c}\text { Second Treatment } \\
\text { Planning Session }\end{array}$ \\
\hline Teeth needing & & \\
$\quad$ Periodontal surgery & 18 & 24 \\
$\quad$ Extraction & 6 & 0 \\
Teeth & & 0 \\
$\quad$ Lost (extracted) & & 0 \\
$\quad$ Saved from extraction & 24 & 18 \\
Tooth status & & \\
\hline
\end{tabular}

teeth had improved to the extent that they were no longer scheduled for extraction. This situation was addressed by creating two additional clinical variables, teeth saved and tooth status. Teeth saved were those formerly hopeless teeth that no longer needed extraction at the second treatment planning session and did or did not require periodontal surgery. Tooth status was defined as teeth needing periodontal surgery, plus hopeless teeth needing extraction, plus teeth lost (extracted during treatment because of symptomatology), minus teeth saved. The tooth status of the patient described above at the first treatment planning session would be 24 teeth; i.e., 18 teeth needing surgery plus 6 teeth needing extraction, whereas at the second treatment planning session, the tooth status would be 18 teeth; i.e., 24 teeth needing surgery minus 6 teeth that were saved from extraction. This is shown in Table 1 . Tooth status thus captured the beneficial effect of saving the teeth from extraction.

It was essential that the periodontist making these clinical decisions be kept double-blind regarding which treatment group the patient was in. This was assured by not breaking the treatment codes until 1985 and then only for purposes of grant reporting. Even then, this examiner was not told which treatment group the patient was in, unless the patient's periodontal health had deteriorated to the extent that retreatment was necessary. As a further precaution to re- 
duce bias by this examiner, he was not directly involved in the treatment of the patients.

\section{Surgery and Recall Phase}

Based on decisions of the clinical examiner, the patient went on to have the necessary periodontal surgery and/or extraction of hopeless teeth, or went directly to the 3-month recall maintenance program (Fig. 1). Patients who completed their surgical treatments were then treatment planned at annual intervals during the recall program by the same examiner. Any needed treatments such as reinstrumentation (scaling/root planing), periodontal surgery, or extractions were recorded and promptly performed while retaining the patients in the recall program. This clinical examiner was blinded as to the patient's treatment group throughout this recall interval.

\section{Bacteriology}

Subgingival plaque was removed from one periodontal pocket per quadrant in each patient except for quadrants that had no teeth or no teeth with pockets $\geq 4 \mathrm{~mm}$. The sites chosen ( 2 to 4 sites per patient) appeared from examination of radiographs to be the most severely involved in each quadrant and were usually around molar teeth. The supragingival plaque about the sample site was removed with a curet and discarded. The subgingival plaque was removed and transferred to a vial containing $0.5 \mathrm{ml}$ of reduced transport fluid (RTF) without EDTA. ${ }^{1}$ The plaque sample was immediately placed within an anaerobic chamber and after dispersing for 20 seconds with a Vortex mixer, a $50 \mu \mathrm{l}$ aliquot was removed for microscopic examination. The remaining sample was diluted to $4 \mathrm{ml}$, sonically dispersed for $20 \mathrm{sec}-$ onds with a Kontes sonifier, ${ }^{\ddagger}$ serially diluted in RTF, and plated automatically with a spiral plater $^{\S}$ on a variety of selective and nonselective media. The method enabled us to obtain microscopic and cultural data on the same plaque sample.

The total anaerobic count, the count of black pigmented bacteroides (BPB) species including Porphyromonas gingivalis, Prevotella intermedia, and Prevotella (B.) melaninogenica, Capnocytophaga species, Fusobacterium nucleatum, and Actinomyces odontolyticus were obtained by identification of their distinctive colonies on enriched trypticase soy agar (ETSA). ${ }^{21}$ Either all or representative colonies of BPB were identified as $P$. gingivalis, $P$. intermedia, and $P(B)$. melaninogenica by a rapid test using chromogenic enzyme substrates. ${ }^{22}$ Actinomyces viscosus and Actinomyces naeslundii were differentiated by catalase activity of colonies growing on a selective GMC agar. ${ }^{23}$ The total count of facultative organisms, Streptococcus sanguis, and Streptococcus mutans was obtained from growth on ETSA agar containing $2 \%$ sucrose and $20 \mu \mathrm{g} / \mathrm{ml}$ of metronidazole, which was incubated anaerobically. Veillonella

\footnotetext{
${ }^{\ddagger}$ Kontes Glass Co., Vineland, NJ.
}

${ }^{\S}$ Spiral Systems, Inc., Cincinnati, $\mathrm{OH}$. colonies were enumerated on a medium containing MM10 base, minus blood but supplemented with lactate, vancomycin, and a $0.004 \%$ bromocresol purple indicator.

Ten $\mu l$ of the aliquot removed for microscopic examination was placed on a glass slide, covered with a $22 \times$ $30 \mathrm{~mm}$ cover slip, sealed, and viewed by dark-field microscopy. Either 200 organisms or the number or organisms in 20 high power fields (hpf) were enumerated, depending on which event occurred first. The single cells were identified as spirochetes, selenomonads, motile rods, fusiforms, nonmotile rods, or cocci.

\section{Statistical Analysis}

The site specific differences in probing depths and attachment levels between the first and second treatment planning sessions were stratified according to the initial pocket depth and averaged per patient. Subsequently, changes in probing depths and attachment levels of pockets initially 1 to $3 \mathrm{~mm}$, 4 to $6 \mathrm{~mm}$ and $\geq 7 \mathrm{~mm}$ per patient were analyzed with a weighted two-factor analysis of variance (ANOVA) (one factor being treatment, the other gender). The number of sites within each disease category ranged considerably from patient to patient. To obtain the best linear unbiased estimates of changes in attachment level and probing depths, all mean patient values were weighted by the inverse of the variance of the patient means. This methodology has been described by Osborn. ${ }^{24}$

Tooth status is an index based on counts of events (i.e., surgeries, extractions, number of teeth saved). This index is unrelated to the number of teeth at risk for such events. As a result, the validity of comparisons across different groups is dependent on similarity of the distribution of the number of teeth at risk across the compared groups. Preliminary analyses showed that the number of teeth at risk in the metronidazole group, and the placebo group, are not significantly different $(P=0.93)$. The mean number of teeth at risk in the metronidazole group and the placebo group was 23.8 (S.D. 2.7) and 23.7 (S.D. 2.7) respectively. We calculated also the percentage of teeth requiring surgery or extraction for each patient at baseline and at the reevaluation stage. The change in tooth status, number of surgical units, and percentage of teeth requiring surgery between the metronidazole and placebo group, were compared with a two-sample $t$-test.

The microscopic findings required a different statistical strategy because of the clustering of responses within a patient and the fact that the various treatments in some individuals reduced the measured parameters below the limits where proportions could be reliably determined. The proportions of the organisms in the microscopic count were calculated by dividing the count of the specific morphotypes in $20 \mathrm{hpf}$ by the total microscopic count in the $20 \mathrm{hpf}$. If less than 20 bacteria were present in the $20 \mathrm{hpf}$, no proportions were calculated. ${ }^{1}$ This procedure eliminated distortion in the data which can occur when the denominator is very low as is sometimes the case after debriding of the 
Table 2: Effect of Scaling, Root Planing and Either Metronidazole or Placebo Upon Surgical Needs of Patients

\begin{tabular}{|c|c|c|c|c|c|c|c|c|c|}
\hline \multirow[t]{2}{*}{$\begin{array}{l}\text { Treatment Group } \\
\text { (number patients) }\end{array}$} & \multicolumn{3}{|c|}{ 1st Treatment Planning Session } & \multicolumn{3}{|c|}{ 2nd Treatment Planning Session } & \multicolumn{3}{|c|}{ Change in } \\
\hline & Surgery* & Status $\dagger$ & $\%$ SU† & Surgery & Status & $\% \mathrm{SU}$ & Surgery & Status & $\% \mathrm{SU}$ \\
\hline Metronidazole (18) & $16.5 \pm 5.6$ & $18.3 \pm 6.0$ & $77 \pm 25$ & $10.3 \pm 6.9$ & $10.0 \pm 6.5$ & $46 \pm 76$ & $6.2 \pm 6.7 \S$ & $8.3 \pm 6.8 \S$ & $31 \pm 28 \S$ \\
\hline
\end{tabular}

*number of teeth needing surgery (mean \pm standard deviation).

†tooth status (mean \pm standard deviation).

$\ddagger \mathrm{SU}$ is surgical unit; \% SU is \% teeth requiring surgery (mean \pm standard deviation).

$\S$ significant difference $P \leq 0.05$ two-sample $t$ test.

Table 3: Change in Probing Depth and Attachment Levels as a Result of Treatment

\begin{tabular}{|c|c|c|c|c|c|}
\hline \multirow{3}{*}{$\begin{array}{l}\text { Initial } \\
\text { Pocket Depth }\end{array}$} & \multicolumn{2}{|c|}{ Debridement plus } & \multirow{3}{*}{$\begin{array}{l}\text { Standard Error } \\
\text { of Difference* }\end{array}$} & \multirow{2}{*}{\multicolumn{2}{|c|}{ Variance Components }} \\
\hline & \multirow{2}{*}{$\begin{array}{c}\text { Metronidazole } \\
(\mathrm{n}=17)\end{array}$} & \multirow{2}{*}{$\begin{array}{l}\text { Placebo } \\
(\mathrm{n}=19)\end{array}$} & & & \\
\hline & & & & $\mathrm{S}_{1}{ }^{2+}$ & $\mathrm{S}_{2}{ }^{2 \dagger}$ \\
\hline \multicolumn{6}{|l|}{$\leq 3 \mathrm{~mm}(22-92)^{\ddagger}$} \\
\hline Probing depth & $-0.05 \mathrm{~mm}^{\S}$ & $0.02 \mathrm{~mm}$ & 0.08 & 0.12 & 0.59 \\
\hline Attachment level & -0.05 & $-0.15^{\S}$ & 0.09 & 0.21 & 1.03 \\
\hline \multicolumn{6}{|l|}{$4-6 \mathrm{~mm}(23-74)$} \\
\hline Probing depth & 0.75 & 0.81 & 0.19 & 0.27 & 1.15 \\
\hline Attachment level & 0.40 & 0.27 & 0.16 & 0.19 & 1.69 \\
\hline \multicolumn{6}{|l|}{$\geq 7 \mathrm{~mm}(1-47)$} \\
\hline Probing depth & 1.91 & 1.50 & 0.30 & 0.62 & 2.08 \\
\hline Attachment level & 0.86 & 0.54 & 0.26 & 0.37 & 2.49 \\
\hline
\end{tabular}

*Adjusted for gender.

${ }^{\dagger} S_{1}{ }^{2}=$ estimate of variance component of patients within treatment group; $S_{2}{ }^{2}=$ estimate of variance component of sites within patient.

${ }^{\ddagger}$ Range of the number of sites present in a patient for the specified category.

${ }^{\S}$ Negative value means either increase in probing depth or loss of attachment.

tooth surfaces. This meant that no post-debridement proportions were calculated in about $20 \%$ of the plaques in which too few bacteria could be observed microscopically (detection limit was about 250,000 colony forming units [cfu]). This problem was not encountered with the cultural counts as the dilution sequence detected as few as $200 \mathrm{cfu}$ in the plaque sample.

Since the presence of low numbers of bacteria is obviously a good outcome, this information should be included in the analysis. For this purpose, the site specific response was labeled as either a success or a failure. For instance, for spirochetes a site was considered successful if a decrease in proportions greater than $5 \%$ occurred, or if the number of bacteria was so low that no proportions could be calculated. A failed site would be one in which the spirochetal count increased or showed $\leq 5 \%$ decrease. The proportions were modeled using modified logistic regression equation. ${ }^{25}$

\section{RESULTS}

All patients reported taking the medication as instructed with the exception of one female in the metronidazole group, who stopped taking the medication after 4 days because of a "leg cramp." Otherwise there were no complaints about the medication. No evidence of the emergence of antibiotic resistant anaerobic organisms was observed in the plaque samples during the course of this investigation. Eighteen metronidazole patients ( 9 male, 9 female; age $47.9 \pm 9.2$ years) and 21 positive control patients (11 male, $10 \mathrm{fe}$ males; age $48.3 \pm 11.5$ years) were available for the first and second treatment planning sessions.

The metronidazole patients had 16.5 teeth that needed periodontal surgery and a tooth status of 18.3 at the first treatment planning session, and these values decreased significantly to 10.3 and 10.0 respectively at the second treatment planning session (Table 2). The placebo patients had 14.2 teeth needing surgery and a tooth status of 15.5 at the first treatment planning session and these values decreased to 12.0 and 12.6 respectively at the second treatment planning session. A two-sample $t$-test for change in tooth status, number of teeth needing surgery, and percent decrease in surgical units (SU) showed that the reduced need for surgery in the metronidazole patients was significant at $P<$ 0.05 (Table 2).

The treatment groups were compared for changes in probing depths and attachment levels. All patients showed a reduction in probing depths and an apparent increase in attachment levels. No significant differences between the treatment groups were observed (Table 3 ). The metronidazole patients tended to exhibit more attachment level gain and pocket reduction than the placebo patients in pockets initially $\geq 7 \mathrm{~mm}$.

Metronidazole treatment was apparently more effective in reducing the need for periodontal surgery than it was to 
Table 4: Relationship Between Clinical Determination of Need for Periodontal Surgery and Probing Depth and Attachment Loss in Teeth That Were Monitored for Bacteriological Changes

\begin{tabular}{lllc}
\hline $\begin{array}{l}\text { First Treatment } \\
\text { Planning } \dagger\end{array}$ & $\begin{array}{c}\text { Probing } \\
\text { Depth }\end{array}$ & $\begin{array}{c}\text { Attachment } \\
\text { Loss }\end{array}$ & $\begin{array}{c}\text { Number of } \\
\text { Teeth }\end{array}$ \\
\hline $\begin{array}{l}\text { Teeth recommended } \\
\text { for surgery }\end{array}$ & $6.3 \mathrm{~mm}$ & $6.4 \mathrm{~mm}$ & 204 \\
$\begin{array}{l}\text { Teeth not recommended } \\
\text { for surgery }\end{array}$ & $\begin{array}{l}4.8 \\
P=0.01 \ddagger\end{array}$ & $\begin{array}{c}5.2 \\
P=0.01\end{array}$ & $\begin{array}{r}99 \\
\end{array}$ \\
& & &
\end{tabular}

Second Treatment

Planning*

Teeth recommended for surgery

Teeth not recommended for surgery

$\begin{array}{lll}5.4 \mathrm{~mm} & 6.2 \mathrm{~mm} & 173 \\ 4.2 & 5.0 & 120 \\ P=.001 & P=0.001 & 293\end{array}$

*After scaling, root planing and medication; 10 teeth had been extracted for acute symptomatology.

$\dagger$ Prior to any treatment.

$\ddagger P$ values obtained from a two-factor analysis of variance.

reducing probing depths and increasing apparent attachment gain. This caused us to examine in greater detail the clinical parameters associated with teeth that did or did not need surgery. For this analysis we used the bacteriological teeth from this study and from an ongoing study; i.e., those teeth selected for bacteriological analysis on the basis of being the most diseased tooth in each quadrant. At the first treatment planning session, 204 of these teeth were recommended for periodontal surgery or extraction. These "surgery" teeth had an average probing depth of $6.3 \mathrm{~mm}$ and an average attachment loss of $6.4 \mathrm{~mm}$, both values were significantly greater than the corresponding values found in the 99 teeth that were not recommended for surgery (Table 4). At the second treatment planning session, the probing depths and the apparent attachment loss decreased for both groups of teeth. However, the teeth recommended for surgery still had significantly higher values than those observed in the teeth not recommended for surgery.

We were interested as to whether this clinical decision could be related to the bacteriological findings. The teeth needing surgery had significantly more bacteria and spirochetes per high power field than did the teeth not needing surgery; i.e., 12.8 bacteria/hpf versus 9.2 bacteria/hpf ( $P$ $=0.0002$, two factor ANOVA), and 8.3 spirochetes $/ \mathrm{hpf}$ versus 5.9 spirochetes/hpf $(P=0.005$, two factor ANOVA). None of the other monitored bacteriological parameters exhibited any significant differences between the teeth needing and not needing surgery.

The reduction in surgical needs in the metronidazole patients could simply have been surgery delayed to the following year. Thus it was important to track the patients during the recall program to determine what surgical needs would arise. Following the second treatment planning session all patients returned to the graduate clinic and received all the indicated surgery and/or extractions and then entered the recall program with a tooth status of 0 . Approximately
Table 5: Treatment Needs of Patients Seen at Recall

\begin{tabular}{|c|c|c|}
\hline & Metronidazole & Positive Control \\
\hline \multicolumn{3}{|c|}{ 3rd Treatment Planning } \\
\hline Number of patients & 14 & 16 \\
\hline Time since medication & $22(16-32)^{*}$ & $25(16-34)^{*}$ \\
\hline Time since hygiene phase & $17(11-27)^{*}$ & $20(13-29)^{*}$ \\
\hline \multicolumn{3}{|l|}{ Number of teeth needing } \\
\hline Periodontal surgery & $3(1) \dagger$ & $3(1) \dagger$ \\
\hline Extraction & $1(1) \dagger$ & 0 \\
\hline Root planing & $49(7) \dagger$ & $54(9) \dagger$ \\
\hline \multicolumn{3}{|c|}{ 4th Treatment Planning } \\
\hline Number of patients & 13 & 15 \\
\hline Time since medication & $34(29-44)^{*}$ & $35(27-42)^{*}$ \\
\hline Time since hygiene phase & $29(25-39)^{*}$ & $30(22-39)^{*}$ \\
\hline \multicolumn{3}{|l|}{ Number of teeth needing } \\
\hline Periodontal surgery & $2(1) \dagger$ & $9(4) \dagger$ \\
\hline Extraction & $2(1) \dagger$ & $14(4) \dagger$ \\
\hline Root planing & $56(7) \dagger$ & $37(4) \dagger$ \\
\hline \multicolumn{3}{|c|}{ Total Need (3rd plus 4th Treatment Planning) } \\
\hline \multicolumn{3}{|l|}{ Number of teeth needing } \\
\hline Periodontal surgery & $5(2) \dagger$ & $12(7) \dagger$ \\
\hline Root planing & $105(9) \dagger$ & $91(10) \dagger$ \\
\hline Extraction & $3(1) \dagger$ & 14 \\
\hline Tooth status (teeth/patient) & 1.91 & 2.77 \\
\hline
\end{tabular}

*Range of months.

$\dagger$ Number of patients.

18 months later one patient in the metronidazole group had 3 teeth in need of surgery and 7 patients had a total of 49 teeth needing root planing (debrided). The corresponding values for the positive control patients were 3 teeth in one patient needing surgery and 54 teeth in 9 patients needing root planing (Table 5). One patient's periodontal condition had deteriorated to the extent that the treatment code was broken and, after determining that she was in the positivecontrol group, she was treated with metronidazole and exited from the formal study, but retained in the recall program. Otherwise all patients received the indicated treatments and reentered into the 3-month recall program.

At the fourth treatment planning session which occurred about 2.5 years after the second treatment planning session, one metronidazole patient had 2 teeth in need of periodontal surgery and 2 teeth in need of extraction, whereas 4 positive-control patients had a total of 23 teeth in need of either surgery or extraction (Table 5). Two of these patients were judged as having relapsed, and after breaking of the code, they were found to be in the positive-control group. They were treated with metronidazole and exited from the formal study, but retained in the recall program. More detailed information on the clinical and bacteriological parameters of these patients will be presented in a separate publication.

The bacteriological data were analyzed to determine what changes, if any, in the plaque flora corresponded with the clinical outcomes. At baseline no differences were observed between the patient groups in any of the bacteriological parameters (Tables 6 and 7). Spirochetes averaged 53\% of the flora in the metronidazole group and $59 \%$ of the flora in the positive control group (Table 6). P. intermedia com- 
Table 6: Effect of Medication and Debridement on Proportions of Bacterial Morphotypes in Subgingival Plaques of Periodontally Involved Teeth

\begin{tabular}{|c|c|c|c|c|c|c|}
\hline & \multicolumn{2}{|c|}{ First Treatment Planning Session } & \multicolumn{2}{|c|}{ After Medication $\dagger$} & \multicolumn{2}{|c|}{ Second Treatment Planning Sessiont } \\
\hline & $\begin{array}{l}\text { Metronidazole } \\
(\mathrm{n}=18 \ddagger)\end{array}$ & $\begin{array}{c}\text { Positive } \\
\text { Control } \\
(\mathrm{n}=21)\end{array}$ & $\begin{array}{c}\text { Metronidazole } \\
(\mathrm{n}=18)\end{array}$ & $\begin{array}{c}\text { Positive } \\
\text { Control } \\
(\mathrm{n}=21)\end{array}$ & $\begin{array}{l}\text { Metronidazole } \\
(\mathrm{n}=18)\end{array}$ & $\begin{array}{c}\text { Positive } \\
\text { Control } \\
(\mathrm{n}=21)\end{array}$ \\
\hline$\%$ Spirochetes & $52.6 \pm 4.1$ & $59.1 \pm 2.5$ & $25.9 \pm 4.3 \|(0.0002)$ & $48.6 \pm 3.5 \|$ & $32.3 \pm 3.2 \|(0.02) \uparrow$ & $47.5 \pm 4.8$ \\
\hline$\%$ Selenomonads & $11.2 \pm 0.8$ & $10.6 \pm 0.9$ & $5.7 \pm 0.9$ & $9.1 \pm 1.1$ & $10.5 \pm 1.0$ & $7.1 \pm 1.0$ \\
\hline \% Vibrio-like & $1.1 \pm 0.3$ & $2.1 \pm 0.5$ & $5.5 \pm 1.3 \|(0.03)$ & $2.2 \pm 0.7$ & $2.2 \pm 0.5$ & $3.0 \pm 1.1$ \\
\hline \% Rods & $18.2 \pm 2.0$ & $16.2 \pm 1.5$ & $19.5 \pm 3.2$ & $14.6 \pm 1.8$ & $27.2 \pm 3.1 \|$ & $21.7 \pm 2.7$ \\
\hline$\%$ Cocci & $6.6 \pm 1.4$ & $4.9 \pm 0.9$ & $24.3 \pm 4.0$ : $(0.02)$ & $11.9 \pm 2.8 \|$ & $10.5 \pm 1.8 \|$ & $11.2 \pm 2.6$ \\
\hline
\end{tabular}

*hpf is microscopic high power field.

$\dagger$ After medication $=1$ to 3 days after completion of unsupervised usage of medication; second treatment planning session is 4 to 6 weeks after completion of all debridement and from 3 to 6 months after usage of medication.

$\ddagger$ Number of patients. The value for each patient is the average of 2 to 4 plaques removed from diseased sites.

§Average \pm standard error.

\|Values are significantly different from baseline values at the first treatment planning session $P \leq 0.01$ paired $t$ test.

TValues in parentheses are the $P$ values determined by the two-sample $t$ test showing significant differences between treatments.

Table 7: Effect of Medication and Debridement on Proportions (average \pm standard error) of Certain Cultivable Species in Subgingival Plaques of Periodontally Involved Teeth

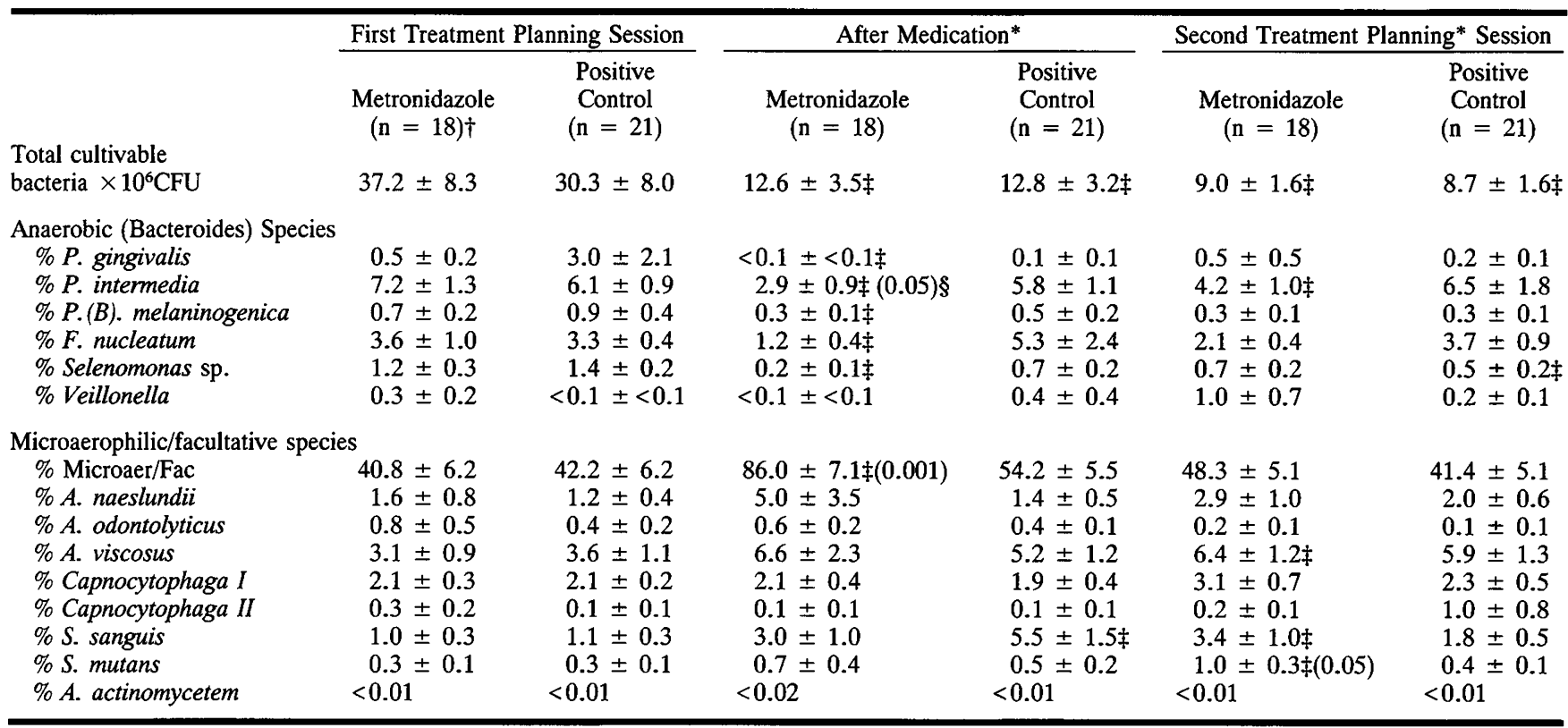

${ }^{*}$ After medication $=1$ to 3 days after completion of unsupervised usage of medication; second treatment planning session is 4 to 6 weeks after completion of all debridement and from 3 to 6 months after usage of medication.

†Number of patients. The value for each patient is the average of 2 to 4 plaques removed from diseased sites.

$\ddagger$ Values are significantly different from baseline values at the first treatment planning session $P \leq 0.05$ paired $t$ test.

$\S$ Values in parentheses are the $P$ values determined by the two-sample $t$ test using subject not sites, which show significant differences between treatment groups.

prised $6 \%$ to $7 \%$ of the cultivable flora, whereas $P$. gingivalis was a minor organism and $A$. actinomycetemcomitans was rarely detected (Table 7). The repeated applications of both paired and unpaired $t$-tests, as was done in Tables 6 and 7, inflates the Type I error rate.

Immediately following medication there was a significant reduction in the total number of organisms in both groups, but the difference between groups was not significant (Ta- bles 6 and 7). In the metronidazole group, there was a significant decrease in the numbers and proportions of spirochetes, in the proportions of selenomonads, motile rods, $P$. gingivalis, $P$. intermedia, $P$.(B). melaninogenica, $F$. nucleatum, and Selenomonas species (all anaerobic organisms), and a significant increase in the proportions of cocci, vibrio-like rods and facultative organisms in the plaques relative to the base line values. In the positive-control group, 
there was a significant decrease in the proportions of spirochetes and significant increase in the proportions of cocci, fusiforms and Streptococcus sanguis. The magnitude of reduction of the anaerobic species was greater in the metronidazole group and this was reflected by significant differences between the treatment groups related to the spirochetes, and the other motile organisms (Table 6) and $P$. intermedia (Table 7). This was balanced by a significant increase in the proportions of facultative/microaerophilic organisms and by cocci and vibrio-like organisms.

The second treatment planning session occurred about 5 months after the medication was taken and 4 to 6 weeks after debridement was completed. At this time in the metronidazole group the proportions and numbers of spirochetes and the proportions of $P$. intermedia were significantly reduced from baseline values, and the proportions of fusiforms, rods, cocci, $A$. viscosus, $S$. sanguis, and $S$. mutans were significantly increased from baseline values (Tables 6 and 7). In the positive-control group, the proportions of spirochetes and Selenomonas sp were significantly decreased from baseline values. Both groups still had appreciable proportions of spirochetes in their plaques $(32 \%$ of the flora in the metronidazole group and $47 \%$ of the flora in the positive-control group). The only significant differences between the treatment groups was a decrease in the spirochetes, and an increase in the fusiforms and $S$. mutans in the metronidazole group (Tables 6 and 7).

The low numbers of bacteria in certain plaque samples after medication and/or debridement precluded the reliable calculation of proportions of the various morphotypes seen in the microscopic examination. This situation was assessed by estimating the frequency with which the proportions or numbers of the various bacterial morphotypes either decreased or increased as a result of treatment. Only the spirochetes exhibited a significant decrease as a result of metronidazole treatment. Thus, $90 \%$ of the monitored metronidazole plus debridement bacteriologic sites at the second treatment planning session showed a reduction in spirochetes in their subgingival plaques, whereas $64 \%$ of the placebo plus debridement sites showed a reduction. This difference between treatment groups was significant $(P<$ 0.05 ). No significant differences were found for the other monitored organisms using this method of analysis.

\section{DISCUSSIONS}

Periodontal patients are concerned mainly with retaining teeth and with the magnitude and duration of the treatment. Any regimen which seeks to improve upon current periodontal therapies should address these concerns. Periodontal surgery is often a recommended treatment for AP and in a high percentage of individuals this acts as a disincentive for the patient to continue with therapy. Any procedure which can maintain teeth in the mouth and which can reduce the need for periodontal surgery would seem to be welcomed.

Treatment outcomes in periodontal disease have traditionally been measured as a reduction in probing depths and as a gain in attachment. ${ }^{26-28}$ In a previous double-blind study, we had found that metronidazole treatment significantly reduced probing depths and caused an apparent attachment gain in the deepest pockets. ${ }^{10}$ However, these benefits were relatively transparent, as many of these patients and teeth still received periodontal surgery. This led us to reconsider what should be the treatment outcome in a clinical trial and resulted in the exploration of a reduced need for surgery as an outcome.

The decision as to whether a tooth does or does not need surgery or extraction is routinely used in clinical practice, but apparently no effort has been made to capture the components of this decision in a systematic fashion. The criteria for surgery described in this investigation represent an effort in this regard. There are no problems with this approach from a statistical perspective, provided that the patients are randomly assigned to the treatment groups and the examining periodontist and patient are kept blind as to the medication received. Under these circumstances, any biases in the application of the surgical criteria should be equally distributed in both treatment groups and should not obscure any treatment effect due, in this case, to the metronidazole. In the present investigation, a 1 week treatment of metronidazole plus thorough debridement of the root surfaces was significantly better than placebo plus thorough debridement in reducing the surgical needs of the patients by about 5 teeth per patient (Table 2).

It has been suggested that the use of "surgery" as an outcome variable may be unreliable as a scientific measure due to the large measurement error. Although measurement error is always of concern in clinical trials, the presence of large measurement errors on the outcome variable in double-blind clinical trials does not necessarily affect the validity of the conclusions. Indeed, it can be shown that when the measurement error is equal for the metronidazole group and the positive-control group, the bias in the measured effect will always be towards the null hypothesis, i.e., no significant differences between the treatments. In the present study, the finding of significant differences between the metronidazole group and the positive control group in the presence of large measurement error suggests that the "true" metronidazole effect is larger than the observed one.

Surgery not indicated at the second planning session could possibly be surgery delayed which would have to be performed in subsequent years. This appears not to have been the case since, when the patients were examined 1 to $1 \frac{1}{2}$ years later, and again 2 to $2 \frac{1}{2}$ years later by the same periodontist, he detected no relapse in surgical needs in the patients subsequently identified as having received metronidazole. Thus the reduced need for periodontal surgery and for tooth extractions were stable benefits that had real significance for both the patient and the clinician. In the positive control group, three patients exhibited clinical relapse within $2 \frac{1}{2}$ years and these patients, as part of their retreatment, were given metronidazole.

The traditional methods of measuring clinical improve- 
ments by reduced probing depths and apparent gain in attachment, however, did not seem to reflect this improvement (Table 3). The foremost reason is lack of power of the statistical tests. In the present study, the power of the statistical test to detect a $0.5 \mathrm{~mm}$ change was less than $35 \%$ for pockets deeper than $6 \mathrm{~mm}$. This low power suggests that mean differences between the treatment modalities rather than the $P$-value should be the primary focus of attention. The data presented in Table 3 are weighted proportionally to the number of sites present in each patient. Such analyses provide a more accurate estimate of the treatment effects. Analyses which are not weighted by the number of sites may result in more unstable estimates of the treatment responses, especially in pockets $>6 \mathrm{~mm}$. In fact, when an unweighted analysis was employed for the present data, a significant difference in attachment levels existed in pockets $>6 \mathrm{~mm}$ (data not shown). Thus, our usage of weighted analysis which showed no significant differences between treatment groups was not only more appropriate statistically, but probably kept us from making a Type I error.

The microbiological results suggest that a reduction in the proportions and numbers of spirochetes can be related to reduction in surgical needs seen at the second treatment planning session in the metronidazole patients (Table 6). This is not surprising as these organisms accounted for more than $50 \%$ of the flora in the subgingival plaques of our patients and they are extremely sensitive in vitro to metronidazole $(0.1 \mu \mathrm{g} / \mathrm{ml})^{30}$ (unpublished data). The presence of high levels and/or proportions of spirochetes in the plaques was the criterion that enabled us to diagnose an anaerobic infection in these patients and to recruit them into the study. Studies which use high proportions of spirochetes; i.e., more than $20 \%$ of the plaque flora, as an entry criterion for treatment with metronidazole reported excellent clinical results, ${ }^{10,17,18}$ whereas those studies in which spirochetes accounted for about $10 \%$ to $15 \%$ of the subgingival flora at baseline report equivocal results. ${ }^{31-34}$ Thus the diagnosis of an anaerobic infection would seem to be required, if one were to expect a beneficial effect from the usage of metronidazole.

Plaque from both groups still contained higher than desired numbers and proportions of spirochetes at the second treatment planning session indicating that neither treatment was truly effective in eliminating spirochetes from the plaque. As anaerobes apparently do not become resistant to metronidazole ${ }^{35}$ and the cultivable spirochetes are sensitive to levels as low as $0.1 \mu \mathrm{g} / \mathrm{ml}$ in vitro, the persistence of relatively high proportions of spirochetes suggests that the metronidazole either did not have access to them because they were in the deeper (tooth side) and/or inaccessible (furcations of the root) layers of the plaque, or that insufficient metronidazole was delivered to the pocket environment to inhibit all the anaerobes present, or that the patients did not fully comply with the instructions for the usage of medication. In medicine, it is estimated that patients are only $40 \%$ compliant in using medication which is to be taken 3 times a day ${ }^{29}$ as was the case in the present study.

The dosage used in this investigation will deliver about 2 to $6 \mu \mathrm{g}$ of metronidazole $/ \mathrm{ml}$ of gingival crevicular fluid (GCF), ${ }^{36}$ which is compatible with the recommended blood levels for metronidazole therapy. ${ }^{35,37}$ However, given that the total volume of GCF per day in a periodontal site may be only $0.5 \mathrm{ml},{ }^{38}$ this drug level may not have been adequate to treat the large number of anaerobes; i.e., $10^{8}$ or more, that would be present in any given pocket with a probing depth $\geq 6 \mathrm{~mm}$. Thus it is possible that with the dosage used and, assuming the patients were totally compliant, that the metronidazole that entered the pocket environment was depleted before it came in contact with the anaerobes or spirochetes in the deeper levels of the plaque, or on the inaccessible surfaces of the root.

If this be the case, then certain procedures could be implemented to increase the level of metronidazole that would be available per plaque anaerobe. The most desirable, and one under development, would be the usage of slow-release delivery systems for placing the metronidazole directly within the pocket. Results utilizing two separate delivery systems indicate that plaque spirochetes can be significantly reduced by this methodology. ${ }^{39,40}$ Another approach would be to debride the tooth surfaces prior to the treatment with systemic metronidazole. If such a treatment schedule had been followed in the present investigation, approximately $70 \%$ fewer bacteria would have been present on the tooth surfaces at the time that metronidazole was taken. A double blind clinical trial making this simple temporal adjustment for the placement of metronidazole is ongoing and preliminary examination of the data suggests that additional clinical benefits can be achieved.

Finally, we would like to propose a model to explain why a 1-week treatment with metronidazole could be exerting a beneficial effect that is maintained for several years after the period of antimicrobial usage (Table 5). Such a long-term effect is possible if the initial clinical condition reflected a specific, albeit chronic, anaerobic infection due to the overgrowth, both relative and absolute, of certain bacterial species which synthesize products that directly or indirectly are deleterious to the periodontal tissues. The tissue damage associated with this overgrowth is usually contained from progressing by the host's immune and inflammatory responses, but these responses do not eliminate the infection because of the extracorporal location of the periodontopathic anaerobes on the root surface. Debridement of the root surface can suppress this infection, but does not eliminate it or adequately contain it, unless the debridement is performed on a regular basis. ${ }^{41}$ The addition of metronidazole to the debridement regimen apparently can suppress this infection to such low levels that the host's immune and inflammatory responses are now able to prevent a reoccurrence of the overgrowth by the periodontopathic species. 
Thus, once the anaerobic periodontopathic members are brought to below a certain numerical threshold in the plaque, they do not contribute to further tissue loss as the combination of host defense mechanisms and periodic debridement are adequate to maintain health. If one uses spirochetes as an indicator organism, then it would appear that about 1 to 2 million spirochetes (about 1 to 2 spirochetes/hpf) can be present in the subgingival plaque without being associated with clinical disease. A similar situation exists in the relationship between $S$. mutans and dental decay. A series of clinical studies demonstrated empirically that, when various treatments reduced the salivary levels of the mutans streptococci to below $2.5 \times 10^{5} \mathrm{CFU} / \mathrm{ml}$ of saliva, dental decay was not likely to occur. ${ }^{42,43}$ By analogy then, the metronidazole plus debridement in this investigation reduced the pocket levels of the anaerobic periodontal pathogens to levels that were compatible with clinical health. This beneficial result would persist as long as the balance between host and microbes was in the favor of the host. It becomes, then, an objective of future research to determine what these threshold levels of the periodontopathic organisms are and to direct therapy to achieve these levels.

\section{Acknowledgments}

Drs. James Giordano and Jack Schwarcz aided in the treatment of the patients, Janice Stoll performed the bacteriological analysis, and Carol Gerlach typed the manuscript. Supported by USPHS Grant DE06080 from the National Institute of Dental Research.

\section{REFERENCES}

1. Loesche WJ, Syed SA, Schmidt E, Morrison EC. Bacterial profiles of subgingival plaques in periodontitis. $J$ Periodontol 1985; 56:447456.

2. Slots J, Genco RJ. Black-pigmented Bacteroides species, Capnocytophaga species, and Actinobacillus actinomycetemcomitans in human periodontal disease: Virulence factors in colonization, survival and tissue destruction. J Dent Res 1984; 63:412-421.

3. Lindhe J, Liljenberg B, Listgarten M. Some microbiological and histopathological features of periodontal disease in man. J Periodontol 1980; 51:264-269.

4. Moore WEC. Microbiology of periodontal disease. J Periodont Res 1987; 22:335-341.

5. Dzink JL, Tanner ACR, Haffajee AD, Socransky SS. Gram negative species associated with active destructive lesions. J Clin Periodontol $1985 ; 12: 648-659$.

6. Zambon JJ. Actinobacillus actinomycetemcomitans in human periodontal disease. J Clin Periodontol 1985; 12:1-20.

7. Kornman KS, Robertson PB. Clinical and microbiological evaluation of therapy for juvenile periodontitis. $J$ Periodontol 1985; 56:443446.

8. Slots J, Hafstrom C, Rosling B, Dahlen G. Detection of Actinobacillus actinomycetemcomitans and Bacteroides gingivalis in subgingival smears by the indirect fluorescent-antibody technique. $J$ Periodont Res 1985; 20:613-620.

9. Loesche WJ. The bacterial etiology of periodontal disease: The specific plaque hypothesis. In: Clinical Dentistry, JW Clark, ed. Philadelphia: Harper \& Row; 1987.

10. Loesche WJ, Syed SA, Morrison EC, Kerry GA, Higgins T, Stoll J.
Metronidazole in periodontitis. I. Clinical and bacteriological results after 15 to 30 weeks. J Periodontol 1984; 55:325-335.

11. Goodson JM, Hogan PE, Dunham SL. Clinical responses following periodontal treatment by local drug delivery. $J$ Periodontol 1985; 56(Suppl):81-87.

12. Shinn DLS. Metronidazole in acute ulcerative gingivitis. Lancet 1962; 1:1191.

13. Rosebury T. Microorganisms Indigenous to Man. New York: McGraw-Hill Book Co., 1962; 310-350.

14. Davies RM, Stirland RM. The in vitro sensitivity of oral spirochetes to metronidazole. J Periodont Res 1970; 5:183-186.

15. Tally FP, Goldin BR, Sullivan N, Johnson J, Gorbach SL. Antimicrobial activity of metronidazole in anaerobic bacteria. Antimicrob Agents Chemother. 1978; 13:460-465.

16. Loesche WJ, Syed SA, Laughon B, Stoll J. The bacteriology of acute necrotizing ulcerative gingivitis. J Periodontol 1982; 53:223-230.

17. Joyston-Bechal S, Smales FC, Duckworth R. Effect of metronidazole on chronic periodontal disease in subjects using a topically applied chlorhexidine gel. J Clin Periodontol 1984; 11:53-62.

18. Lindhe J, Liljenberg B, Adielson B, Borjesson I. Use of metronidazole as a probe in the study of human periodontal disease. $J$ Clin Periodontol 1983; 10:100-112.

19. Ramfjord SP, Knowles JW, Nissle RR, Schick RA, Burgett FG. Longitudinal study of periodontal therapy. J Periodontol 1973; 44:6677.

20. Schmidt EF, Webber RL, Ruttimann UE, Loesche WJ. Effect of periodontal therapy on alveolar bone as measured by subtraction radiography. J Periodontol 1988; 59:633-638.

21. Syed SA, Svanberg M, Svanberg G. The predominant cultivable dental plaque flora of beagle dogs with periodontitis. $J$ Clin Periodontol 1981; 8:45-56.

22. Laughon BE, Syed SA, Loesche WJ. Rapid identification of Bacteroides gingivalis. J Clin Microbiol 1982; 15:345-346.

23. Zylber LJ, Jordan HV. Development of a selective medium for detection and enumeration of Actinomyces viscosus and Actinomyces naeslundii in dental plaque. J Clin Microbiol 1982; 15:253-259.

24. Osborn $\mathrm{J}$. The choice of computational unit in the statistical analysis of unbalanced clinical trials. $J$ Clin Periodontol 1987; 14:519-523.

25. Williams DA. Extra-binomial variation in logistic linear models. App Statist 1982; 31:144-148.

26. Knowles JW, Burgett FG, Nissle RR, Shick RA, Morrison EC, Ramfjord S. Results of periodontal treatment related to pocket depth and attachment level. J Periodontol 1979; 50:225-233.

27. Lindhe J, Westfelt E, Nyman S, Socransky SS, Heijl L, Bratthall G. Healing following surgical/nonsurgical treatment of periodontal disease. A clinical study. J Clin Periodontol 1982; 9:115-128.

28. Pihlstrom BL, Oliphant TH, McHugh RB. Molar and nonmolar teeth compared over $61 / 2$ years following two methods of periodontal therapy. $J$ Periodontol 1984; 55:499-504.

29. Greenberg RN. Overview of patient compliance with medication: A literature review. Clin Ther 1984; 6:592-599.

30. Cheng SL, Siboo R, Chin Quee T, Johnson JL, Mayberry WR and Chan ECS. Comparative study of six random oral spirochete isolates. $J$ Periodont Res 1985; 20:602-612.

31. Jenkins WMM, MacFarlane TM, Gilmour WN, Ransay I, MacKenzie D. Systemic metronidazole in the treatment of periodontitis. $J$ Clin Periodontol 1989; 16:443-450.

32. Sterry KA, Langeroudi M, Dolby AE. Metronidazole as an adjunct to periodontal therapy with sub-gingival curettage. Brit Dent J 1985; 158:176-178.

33. Van Oosten MA, Mikx FH, Renggli HH. Microbial and clinical measurements of periodontal pockets during sequential periods of nontreatment, mechanical debridement and metronidazole therapy. J Clin Periodontol 1987; 14:197-204.

34. Watts T, Palmer R, Floyd P. Metronidazole: A double-blind trial in untreated human periodontal disease. J Clin Periodontol 1986; 13:939 943. 
35. Tally FP, Sutter VL, Finegold SM. Treatment of anaerobic infections with metronidazole. Antimicrob Agents Chemother 1975; 7:672-675.

36. Britt MR, Pohlod DJ. Serum and crevicular fluid concentration after a single oral dose of metronidazole. J Periodontol 1986; 57:104-107.

37. Goldman P. Drug therapy: Metronidazole. $N$ Engl J Med 1980; 303:1212-1218.

38. Goodson JM. Pharmacokinetic principles controlling efficacy of oral therapy. $J$ Dent Res 1989; 68:1625-1632.

39. Loesche WJ, Schmidt E, Smith AA, Caffesse R, Stoll J. Metronidazole therapy for periodontitis. J Periodont Res 1987; 22:224-226.

40. Addy M, Hassan H, Moran J, Wade W, Newcombe R. Use of antimicrobial-containing acrylic strips in the treatment of chronic peri- odontal disease - a three month follow-up study. J Periodontol 1988; 59:557-564.

41. Nyman S, Lindhe J, Rosling B. Periodontal surgery in plaque infected dentitions. J Clin Periodontol 1977; 4:240-249.

42. Emilson CG, Krasse B. Support for and implications of the specific plaque hypothesis. Scand J Dent Res 1985; 93:96-104.

43. Loesche WJ. Role of streptococcus mutans in human dental decay. Microbiol Rev 1986; 50:353-380.

Send reprint requests to Dr. Walter J. Loesche, University of Michigan, School of Dentistry, Ann Arbor, MI 48109-1078.

Accepted for publication October 15, 1990. 